\title{
Cam Drive Step Mechanism of a Quadruped Robot
}

\author{
Qun Sun, Chong Wang, Dongjie Zhao, and Cuihua Zhang \\ School of Mechanical and Automotive Engineering, Liaocheng University, Liaocheng, Shandong 252000, China \\ Correspondence should be addressed to Qun Sun; sunxiaoqun97@163.com
}

Received 5 February 2014; Revised 10 April 2014; Accepted 22 April 2014; Published 10 July 2014

Academic Editor: Shahram Payandeh

Copyright (C) 2014 Qun Sun et al. This is an open access article distributed under the Creative Commons Attribution License, which permits unrestricted use, distribution, and reproduction in any medium, provided the original work is properly cited.

\begin{abstract}
Bionic quadruped robots received considerable worldwide research attention. For a quadruped robot walking with steady paces on a flat terrain, using a cam drive control mechanism instead of servomotors provides theoretical and practical benefits as it reduces the system weight, cost, and control complexities; thus it may be more cost beneficial for some recreational or household applications. This study explores the robot step mechanism including the leg and cam drive control systems based on studying the bone structure and the kinematic step sequences of dog. The design requirements for the cam drive robot legs have been raised, and the mechanical principles of the leg operating mechanism as well as the control parameters have been analyzed. A cam drive control system was constructed using three cams to control each leg. Finally, a four-leg demo robot was manufactured for experiments and it showed stable walking patterns on a flat floor.
\end{abstract}

\section{Introduction}

Quadruped robots have been extensively studied as the most important branch of bionic robot applications. In 1968, General Electrics and the US Army Mobility Systems Laboratory constructed a quadruped walking machine, which used hydraulic server motors to drive [1]. It was later suggested that a large number of controllable degrees of freedom require highly efficient drives properly arranged, special design of feet to dissipate the energy of the strike, and so forth, and the problem of the control seems to be the main problem of the walking robot [2].

The first comprehensive quadruped robot, KUMO-I, was developed in 1976 by Japan's Tokyo Institute of Technology, which subsequently also produced TITAN series quadruped robots based on this study [3-6]. Currently quadruped robots are widely studied in many universities and laboratories around the world [7-13]. The most advanced quadruped robot so far is BigDog, jointly developed by Boston Dynamics and M.I.T. in 2005 for the US Army. BigDog is able to be operated in unstructured environment, with multiple capabilities such as independent walking, running, jumping, and climbing obstacles. An upgraded version, LS3, is currently in intense development [13].

Considered in a constructed environment with flat terrain, the robot performs steady walking, and it is possible to use a mechanical transmission system to replace the servomotors at the leg joints. This reduces the system weight, cost, and control complexities; thus it may be more cost beneficial for some recreational or household applications. For this reason, this study utilizes some previous research outcomes [14-16] and the bionic theories to improve the theoretical analysis and the component design and presents a novel cam drive quadruped robot step mechanism to achieve steady walking on an even floor surface.

\section{Analysis of the Step Sequences of Typical Quadrupeds}

2.1. Kinematic Bone Structure of Typical Quadrupeds. The animals that are most widely used in the bionic studies for quadruped robots are dogs. According to observations and analysis of dog walking, scientists investigated the dogs bone structures involved in body movement and walking, including the shoulder, elbow, hip, and knee joints [3].

The dog's shoulder can be regarded as a ball joint, which is able to tilt back and forth or yaw to the two sides, as shown in Figure 1. The dog's elbow is similar to an articulated joint structure, which can only move in a single plane, that is, tilting back and forth, to the maximum 90-degree angle as presented in Figure 2. Hip is another ball joint structure 


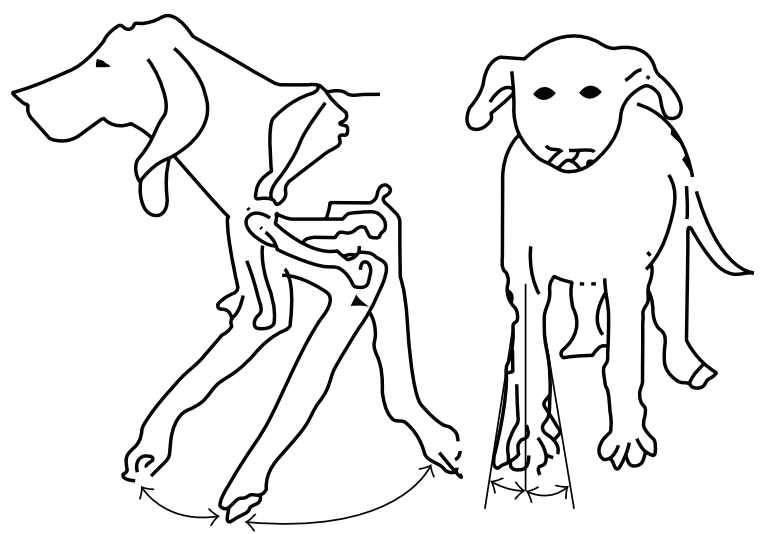

Figure 1: Movements of dog's shoulder.

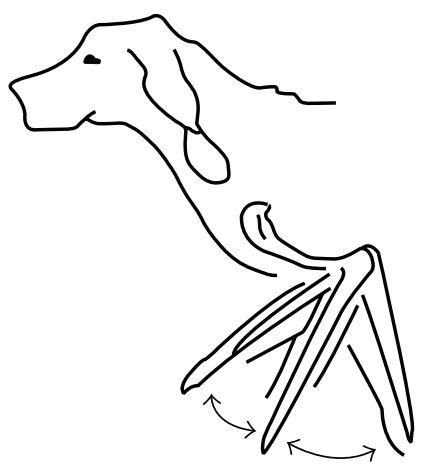

Figure 2: Movements of dog's elbow.

which can turn about three axes, as shown in Figure 3. Similar to elbow, knee is another articulated joint structure which can only tilt back and forth, as shown in Figure 4.

2.2. Step Sequence Analysis of Typical Quadrupeds. When quadrupeds steadily walk, the four legs move coordinately and each leg is in turn in two different states. The step sequence usually obeys the diagonal principle; that is, the left front leg moves first and then the right lateral, the left lateral, the right front, the left front leg, and so on in repeated cycles. When a quadruped is in the state of a supporting phase, there are at least three legs touching the ground, whilst in the hanging phase there is only one leg lifted above the ground. Therefore, regarding the motion of each leg in steady walking, the ratio between the time that the foot is on the ground and off ground, that is, the duty cycle, is 0.75 .

The limiting condition of this study is that a quadruped robot walks following a steady and regular pace; that is, a robot moves in a steady manner and changing position is achieved by continuously shifting gaits following the fundamental modes. The step mechanisms described in this paper include leg mechanism and the cam drive control system that governs the motions of the leg joints. These two aspects will be presented in the following sections.

\section{Theoretical Analysis of Robot Leg Mechanism}

3.1. Design Requirements of Leg Mechanism. According to quadruped walking characteristics, the design requirements of the quadruped robot walking mechanism are given below [15].

(1) The movement of the foot end relative to the robot body is of a dome shape $\frown$, where the straight line section is the motion trajectory of the foot supporting mechanism in the supporting phase. The curve section corresponds to the foot trajectory when it is lifted above the ground, that is, in the hanging phase.

(2) In order to avoid unnecessary energy consumption involved in body bumping when the robot moves, it is necessary to ensure certain straightness of the straight line section mentioned in (1).

(3) In order to avoid friction and unnecessary energy consumptions occurring due to uneven speed of three supporting legs when the robot moves, it is proposed to ensure that each leg moves in a steady speed within the straight line section mentioned in (1).

(4) Regarding the curve section in (1), there is a requirement about the ceiling point rather than the shape, since the maximum height determines the ability that the robot travels on an uneven surface.

(5) In addition to requirement (1), the time that a foot travels along the straight line section should be three times of that used in the curved section. This means that the phase angle of the supporting phase is $3 \pi / 2$ whilst for the hanging phase it is $\pi / 2$. The coordinated motion of the four legs should obey the diagonal principle; that is, the difference of the phase angle between the two legs that move in turn should be $\pi / 2$.

3.2. Mechanical Principle of the Leg Mechanism. The scope of this study is the step mechanism of a quadruped robot that can achieve steady walking, excluding turning. By simplifying the real leg joint structure based on the hip, elbow, and knee joints, the mechanical schematic of the leg structure can be shown in Figure 5.

In Figure 5, sections $B C, \mathrm{O}_{2} B$, and $\mathrm{O}_{1} \mathrm{O}_{2}$, points $\mathrm{B}$ and $\mathrm{O}_{2}$ correspond to the calf, thigh, hip bone, knee joint, and hip joint, respectively. The associated parameters are as follows [14-16]: (1) $E$ is hip joint offset gap, (2) $h_{1}$ is calf length, (3) $h_{2}$ is thigh length, (4) $S$ is step length, (5) $\theta_{1}$ is hip joint turning angle, (6) $\theta_{2}$ is step angle, and (7) $\theta_{3}$ is knee joint turning angle. The designed leg parameters are (1) hip joint offset gap $E=50 \mathrm{~mm}$, (2) calf length $h_{1}=220 \mathrm{~mm}$, (3) thigh length $h_{2}=180 \mathrm{~mm}$, and (4) step length $S=150 \mathrm{~mm}$.

The leg mechanism required to achieve the movement described in Figure 5 needs the following structural criteria [15]: firstly assume the starting point is $\theta_{1}=0$ and $\theta_{2}=0$; thus if joint $A$ moves to the right, then the foot $C$ tends to move along a circle whose center is $\mathrm{O}_{2}$ and the radius is $\left(h_{1}+\right.$ $h_{2}$ ). If point $A$ maintains uniform motion in the horizontal 

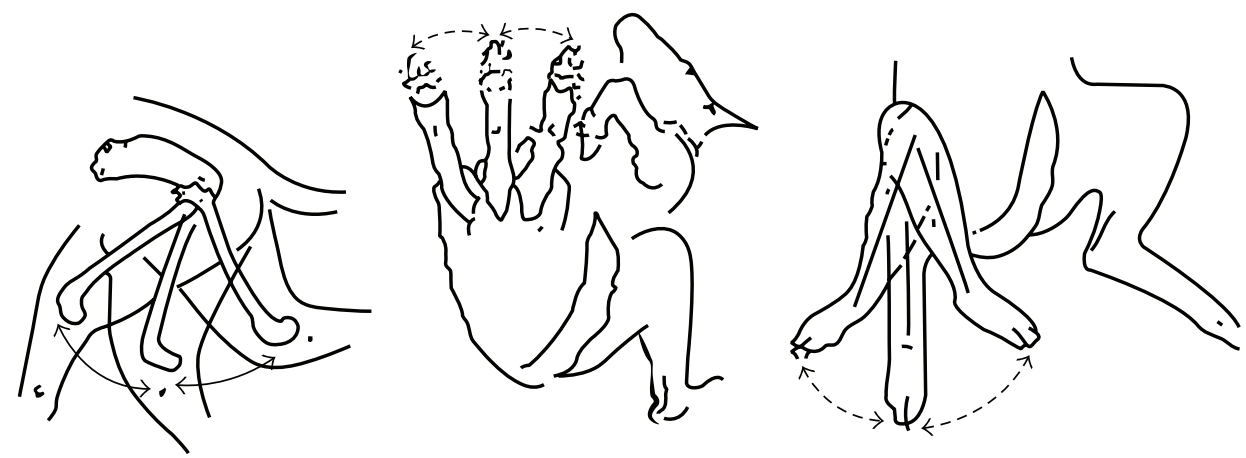

FIGURE 3: Movements of dog's hip.

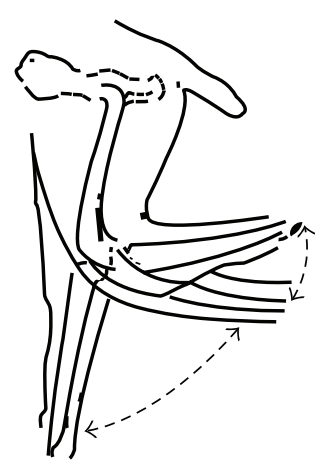

Figure 4: Movements of dog's knee.

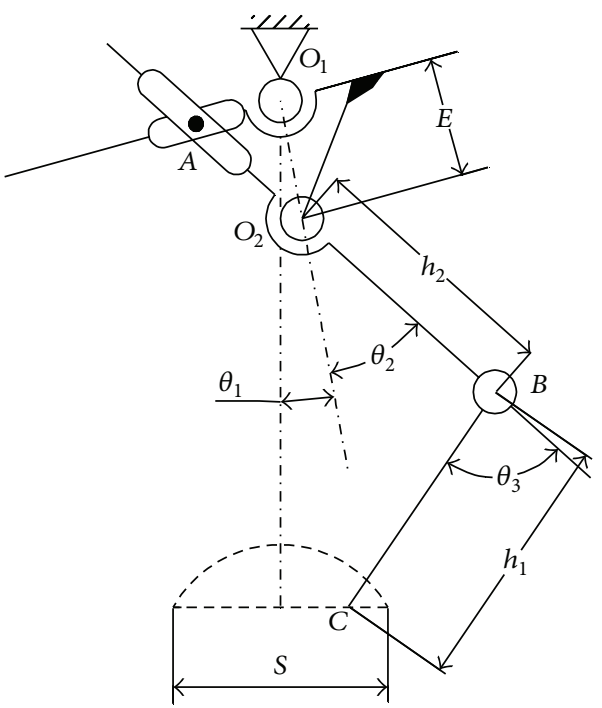

FIGURE 5: Schematic diagram of the walking mechanism.

direction, then the intersection between calf $B C$ and the flat dash line in Figure 5 also maintains steady horizontal velocity. Therefore, if the hip joint angle $\theta_{1}$ and the knee join $\theta_{3}$ turn to enable the foot end $C$ to move to this intersection point, then it satisfies the requirements (2) and (3) mentioned previously in Section 3.1. Next, move joint $A$ quickly to the left, making sure the displacement equals that in the last procedure but the

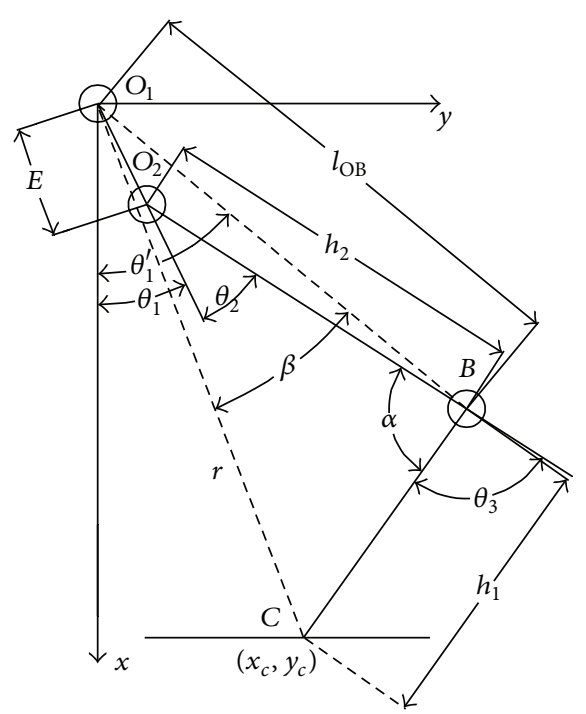

FIGURE 6: Schematic diagram of robot leg in the Cartesian coordinate system.

travelling time is only $1 / 3$ of it and then enable foot end $C$ to move along the curved dash line in Figure 5.

3.3. Joint Parameters and Drive Principle. As shown in Figure 6, in order to satisfy quadruped robot walking requirements, when a cam drive robot leg is in the hanging phase, it is only necessary to control the position of the foot $\left(x_{c}, y_{c}\right)$. In the supporting phase, however, the relationships between the three angles $\theta_{1}, \theta_{2}$, and $\theta_{3}$ should be clearly defined and controlled. [15]:

The parameter $\theta_{2}$ should satisfy the following constraint

$$
\theta_{2}=\arctan \frac{y_{c}}{\sqrt{\left(x_{c}^{2}+y_{c}^{2}\right)-(S / 2)^{2}}}
$$

where $S$ is the step length which is the same as that defined in Figure 5. The relationships between $\theta_{1}$ and $\theta_{3}$ relate to the inverse kinematic solution of the in-plane two-bar linkages 


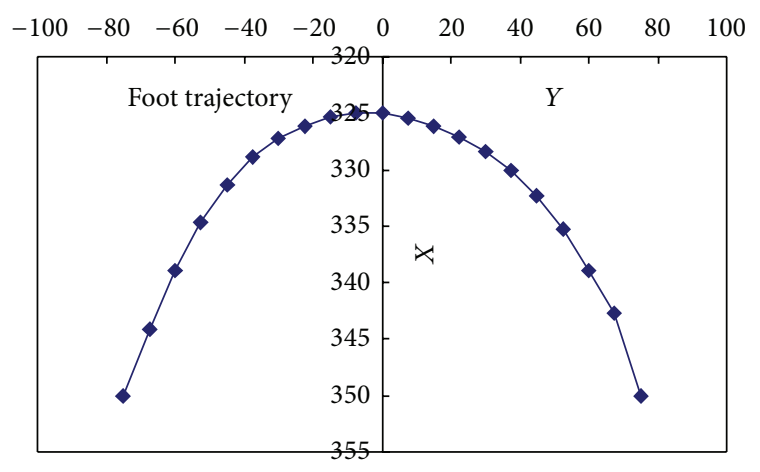

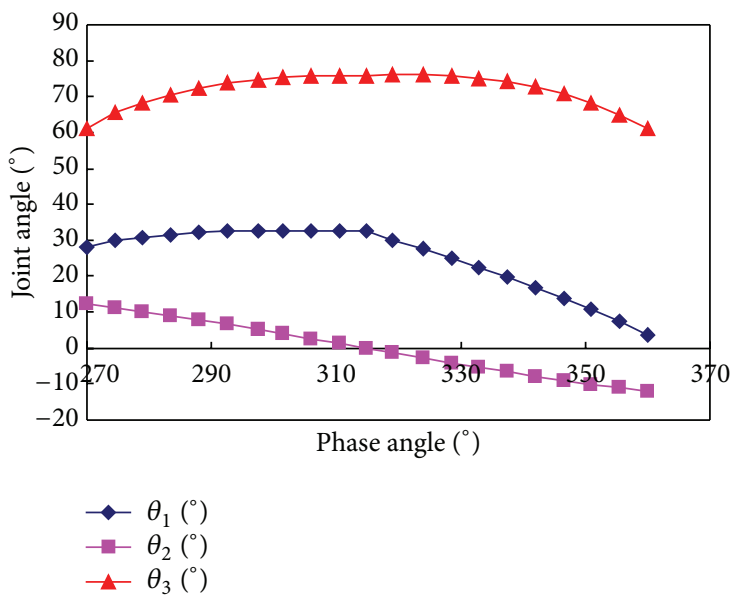

(b)

FIGURE 7: Key sample points chosen for cam control.

$O_{1} B$ and $B C$. By analyzing in-plane geometry, the kinematics functions of the linkage are given by

$$
\begin{aligned}
& x_{c}=l_{\mathrm{OB}} \cos \left(\theta_{1}+\angle O_{2} O_{1} B\right)+h_{1} \cos \left(\theta_{1}+\angle O_{2} O_{1} B-\theta_{3}\right), \\
& y_{c}=l_{\mathrm{OB}} \sin \left(\theta_{1}+\angle O_{2} O_{1} B\right)+h_{1} \sin \left(\theta_{1}+\angle O_{2} O_{1} B-\theta_{3}\right) .
\end{aligned}
$$

The below relationship can be obtained from cosine law:

$$
l_{\mathrm{OB}}^{2}=h_{1}^{2}+E^{2}-2 h_{1} E \cos \angle O_{1} O_{2} B,
$$

and from law of sines the following is given:

$$
l_{\mathrm{OB}} \sin \angle O_{2} O_{1} B=h_{1} \sin \theta_{2} \text {. }
$$

The inverse solution of the in-plane linkage kinematics function is about deriving $\theta_{1}$ and $\theta_{2}$ when $\left(x_{c}, y_{c}\right)$ is given. It is possible to derive

$$
\theta_{1}^{\prime}=\arctan \left(\frac{y}{x}\right) \pm \beta
$$

where

$$
\begin{gathered}
\beta=\arccos \left[\frac{\left(r^{2}+l_{O B}^{2}-h_{2}^{2}\right)}{\left(2 l_{O B} r\right)}\right], \\
r^{2}=x_{c}^{2}+y_{c}^{2} .
\end{gathered}
$$

According to the actual situation of quadruped moving, either the calf and thigh remain in a straight line or the calf is behind the knee. However, since $\theta_{1}^{\prime}=\arctan (y / x)-$ $\beta$ is derived from an ideal theoretical situation without considering this aspect, (5) can be simplified as

$$
\theta_{1}^{\prime}=\arctan \left(\frac{y}{x}\right)+\beta
$$

Hence,

$$
\theta_{1}=\theta_{1}^{\prime}-\angle O_{2} O_{1} B
$$

From

$$
\begin{gathered}
l_{\mathrm{O}_{2} \mathrm{C}}^{2}=\sqrt{\left(X_{c}-X_{\mathrm{O}_{2}}\right)^{2}+\left(Y_{c}-Y_{\mathrm{O}_{2}}\right)^{2}}, \\
X_{\mathrm{O}_{2}}=E \sin \theta_{1}, \\
Y_{\mathrm{O}_{2}}=E \cos \theta_{1},
\end{gathered}
$$

the following can be derived:

$$
\alpha=\arccos \left[\frac{\left(h_{1}^{2}+h_{2}^{2}-l_{\mathrm{O}_{2} \mathrm{C}}^{2}\right)}{\left(2 h_{1} h_{2}\right)}\right] .
$$

Therefore,

$$
\theta_{3}=\pi-\alpha
$$

Equations (1), (8), and (11) form the relationship functions between $\theta_{1}, \theta_{2}$, and $\theta_{3}$. In the hanging phase, to control $\theta_{1}, \theta_{2}$, and $\theta_{3}$, it is only necessary to know a few point coordinates. This paper chooses some key points for control purpose as shown in Figure 7, where each sample point was obtained by equally dividing the total phase angle $\pi / 4$ of a hanging phase.

Given by the current technologies, using either electric, hydraulic, or pure mechanical drive methods can implement the control of $\theta_{1}, \theta_{2}$, and $\theta_{3}$. In order to reduce the cost and control complexities, this paper uses 4 groups of cams to control the 3 joint parameters on each of the 4 legs, totally 12 parameters, which can enable quadruped robot steady walking on an even terrain.

\section{Cam Drive and Control Mechanism of Quadruped Robot}

4.1. The Principle of the Hip Joint Cam Kinematics. The movement principle of the hip joint cam drive control parameter $\theta_{1}$ is shown in Figure 8. 


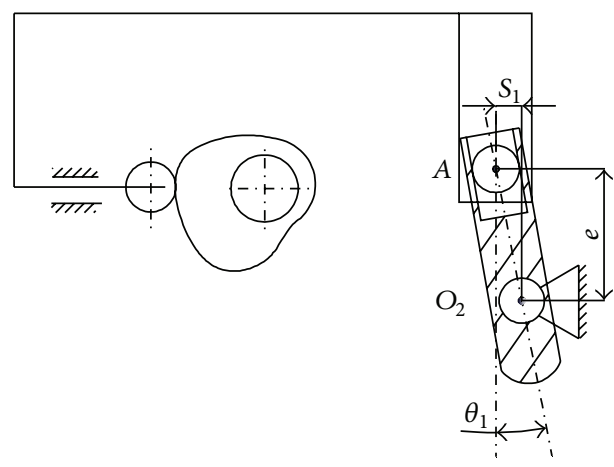

FIGURE 8: The principle of the hip joint cam kinematics.

The moving part of the hip joint cam is subject to the below law of motion:

$$
s_{1}=e \cdot\left[\tan \theta_{1}-\tan \theta_{1}^{*}\right] .
$$

In this paper $e=30$, where $e$ is the vertical distance between points $A$ and $\mathrm{O}_{2}, A$ is a movable pivot point, and $\mathrm{O}_{2}$ is a fixed pivot point. The initial value of $\theta_{1}$ is $\theta_{1}^{*}=0.11768$, and in the hanging phase, the $\theta_{1}^{*}$ values are given in Figure 7 . Therefore, the $(x, y)$ coordinates of the points on the cam profile curve can be written as

$$
\begin{aligned}
& x=\left(r_{o 1}+s_{1}\right) \cos \varphi, \\
& y=\left(r_{o 1}+s_{1}\right) \sin \varphi,
\end{aligned}
$$

where $s_{1}$ is the pushing distance of the hip joint cam follower and $\varphi$ is the rotational angle of the camshaft.

Equation (13) can only provide the theoretical profile curve of the hip joint cam. The actual profile curve is the inner envelop of a series of circles, whose centers are the points on the theoretical profile curve, and the radius is the radius of the rolling wheel. C programming was used to sample 50 points equally from the whole circumference of the cam, and then these points were smoothly connected to produce an approximated cam model.

4.2. The Principle of the Main Cam Kinematics. The principle of the main cam control parameter $\theta_{2}$ is to use the motion of the movable joint $A$ along $A O_{1}$ presented in Figure 5 to govern the follower. The cam structure to $\operatorname{control} \theta_{1}$ is shown in Figure 9.

The stroke length of the main cam is shown in Figure 10.

In Figure 10, $a$ is the sum of the follower stroke and the radius of the cam base plate, when the follower is at the turning point when approaching the angle of repose. And $b$ is the follower stroke and the radius of the cam base plate when the follower is at the angle of repose. In this paper, $a=10.8932$ and $b=13.6771$. The schematic diagram of the main cam follower motion cycle is presented in Figure 11.

In Figure 11, $n$ is the radian angle of the camshaft rotation $\varphi$ and $n=\varphi \pi / 180$. Assume $s_{2}$ is the stroke of the main cam follower, divided into three sections corresponding to $O A$, $A B$, and $B C$. If the cam follower strokes corresponding to

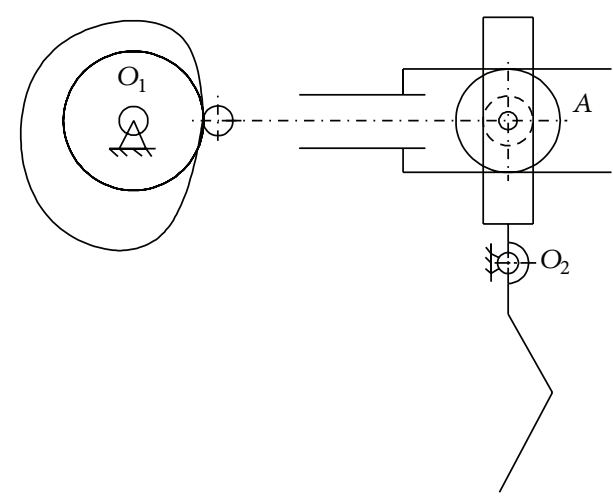

Figure 9: The principle of the main cam kinematics.

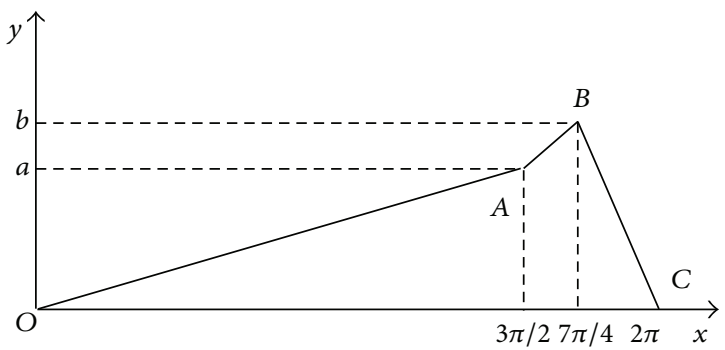

Figure 10: The stroke of the main cam.

sections $O A, A B$, and $B C$ are $s_{21}, s_{22}$, and $s_{23}$, respectively, then the below equations can be given:

$$
\begin{gathered}
s_{21}=\frac{(2 \cdot a \cdot n)}{3 \pi}, \\
s_{22}=\frac{4 \cdot(b-a) \cdot n}{(\pi+7 a-6 b)}, \\
s_{23}=\frac{-4 \cdot b \cdot n}{(\pi+8 b)} .
\end{gathered}
$$

Therefore, the $(x, y)$ coordinates of the points on the cam profile curve can be expressed as

$$
\begin{aligned}
& x=\left(r_{o 2}+s_{2}\right) \cos \varphi, \\
& y=\left(r_{o 2}+s_{2}\right) \sin \varphi .
\end{aligned}
$$

Similarly (15) can only give the theoretical profile curve of the main cam, and $\mathrm{C}$ programming was used to produce a fitted cam model.

4.3. The Principle of the Knee Joint Cam Kinematics. The principle of the knee joint cam control parameter $\theta_{3}$ is given in Figure 12, and the knee joint cam follower follows the below relationship:

$$
\frac{r \theta_{3}}{r_{2}}=\frac{s_{3}}{r_{1}}
$$

which leads to

$$
s_{3}=\frac{r_{1} \theta_{3} r}{r_{2}} \text {. }
$$




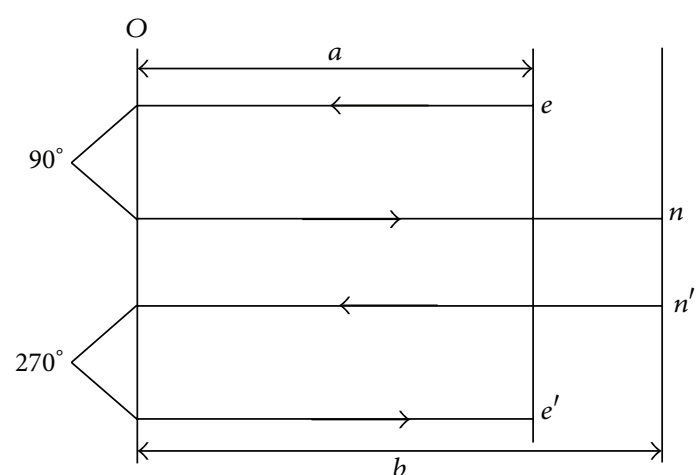

FIGURE 11: Schematic diagram of the main cam motion cycle.

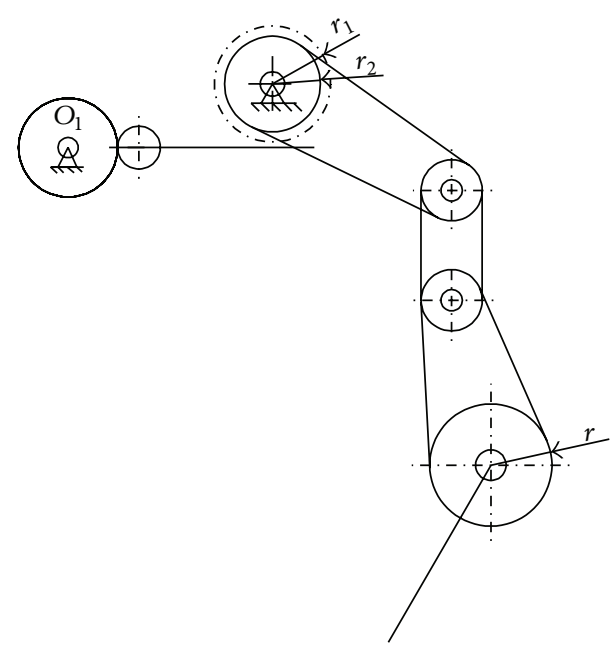

FIGURE 12: The knee joint cam control method.

The $(x, y)$ coordinates of the points on the cam profile curve can be written as

$$
\begin{aligned}
& x=\left(r_{o 3}+s_{3}\right) \cos \varphi, \\
& y=\left(r_{o 3}+s_{3}\right) \sin \varphi,
\end{aligned}
$$

where the $\theta_{3}$ value in the supporting phase is given by (11). In order to verify the strokes of the three joints presented correctly, some key points can be used as shown in Figure 13.

\section{Design of the Quadruped Robot Step Mechanism}

Virtual system design software, Pro/E, was utilized to model the overall three-dimensional structure of the step mechanism of the cam drive quadruped robot, as shown in Figure 14.

The system was assembled and integrated after different components were manufactured. An actual example of the assembled quadruped robot is shown in Figure 15.

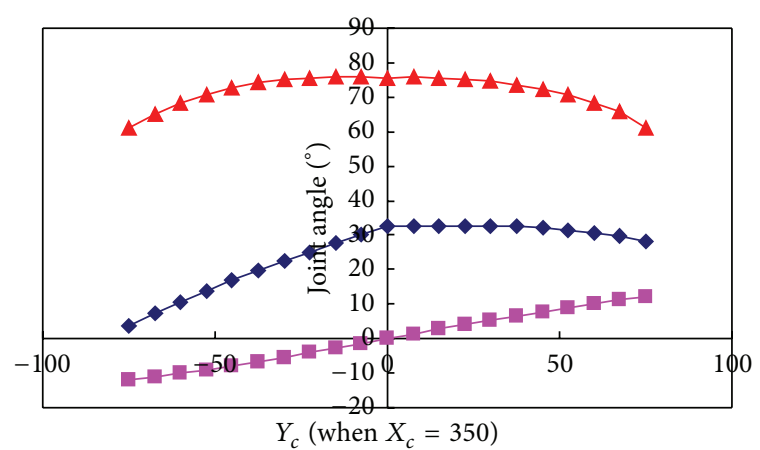

$$
\begin{aligned}
& \leftarrow \theta_{1}\left(^{\circ}\right) \\
& -\theta_{2}\left(^{\circ}\right) \\
& \leftarrow \theta_{3}\left(^{\circ}\right)
\end{aligned}
$$

FIGURE 13: Key sample points chosen for verifying the fitted cam model.

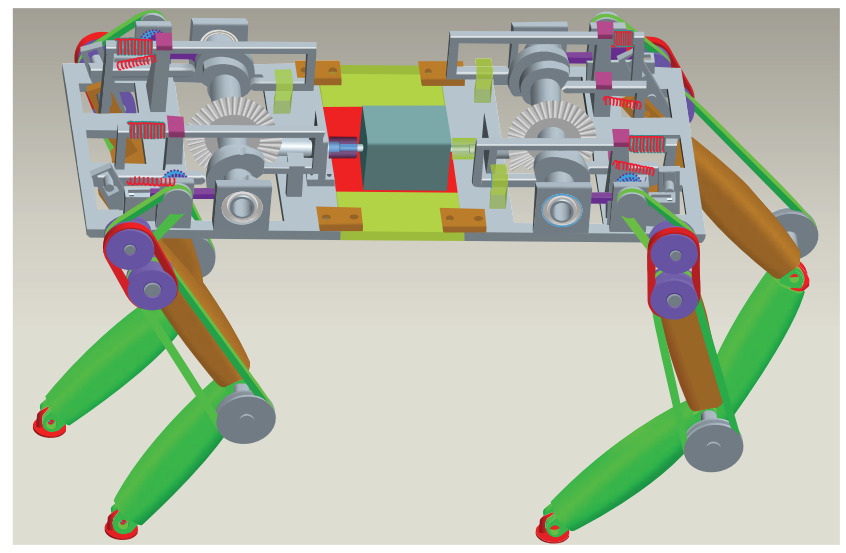

FIGURE 14: Three-dimensional model of the quadruped robot.

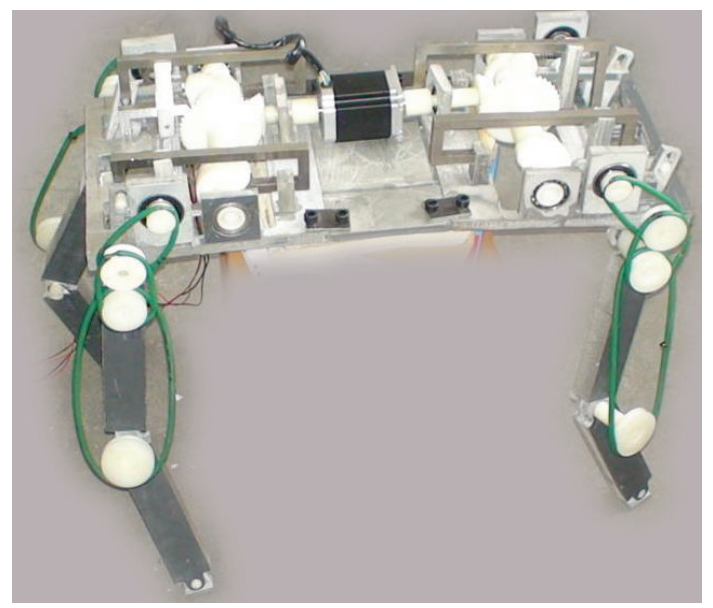

FIGURE 15: An example quadruped robot LCQR-1.

\section{Experiments}

The quadruped robot prototype has been built with a controller that can perform walking tests. As shown in Figure 16, the control system employs an 80C51 MCU, a DAC0832 unit that transfers the digital control command to analog 


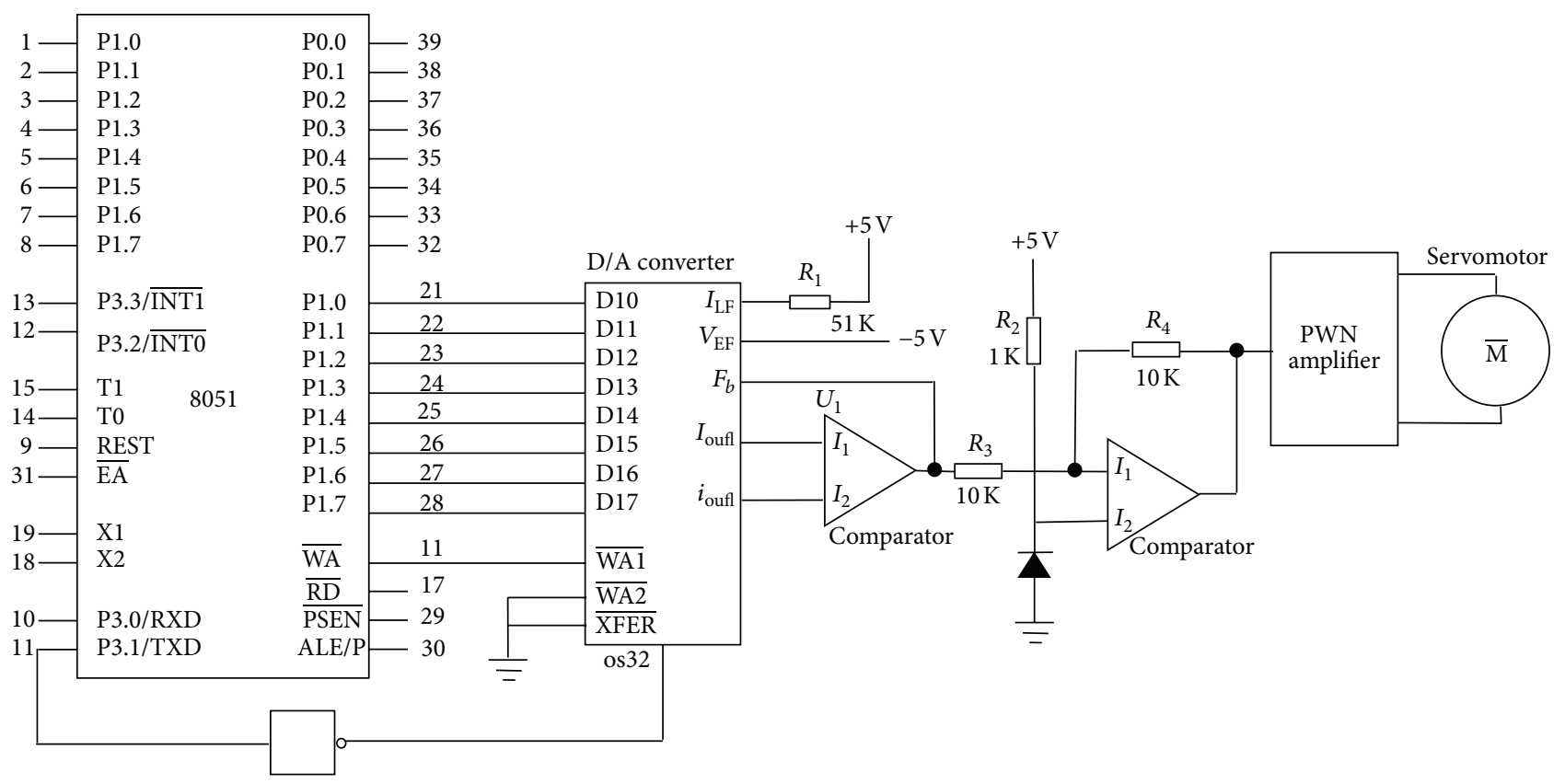

FIGURE 16: The control unit of the prototype quadruped robot.

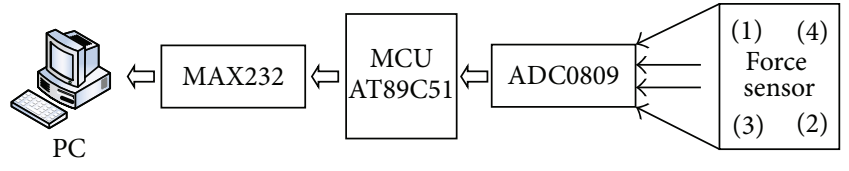

FIGURE 17: The foot supporting force measurement unit.

signals, and a PWN amplifier to drive the servomotor. Since the legs are all driven by the servomotor through the cam mechanisms, only one servomotor is needed, which simply governs the leg paces by motor rotational speed.

In order to assess the walking tests, a force measurement unit was also developed, which consists of a force sensor at the bottom of each foot, a ADC0809 unit that transfers analog force signals into digital data required by the $80 \mathrm{C} 51 \mathrm{MCU}$, and the recorded data were sent to a remote PC through MAX232 interface. The four legs and the sensors are numbered (1)(4) as shown in Figure 17, which are consistent with the quadruped robot step sequence as mentioned previously.

The supporting force of each foot was recorded with $1 \mathrm{KHz}$ sampling rate and then averaged for every twenty samples to smooth out transient disturbances. Some testing results for the robot walking with a speed of two steps per second are shown in Figure 17, where the duty cycle of the step sequence is 0.75 ; that is, when one leg is lifted above the ground, all the other three legs are in the supporting phase. As the legs are all driven by one servomotor and a set of mechanical linkages, they do appear to hit the ground and leave the ground in a somehow rigid manner; there are clear force distinctions between the supporting phase and the hanging phase.

As shown in Figure 18, the force measured at the bottom of each foot is zero in the hanging phase and is about

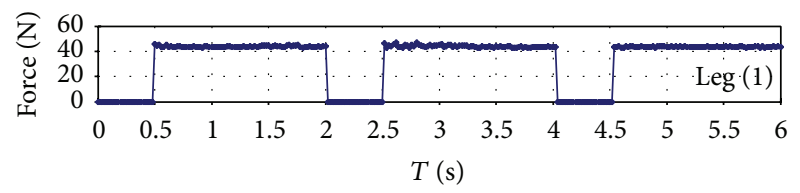

(a)

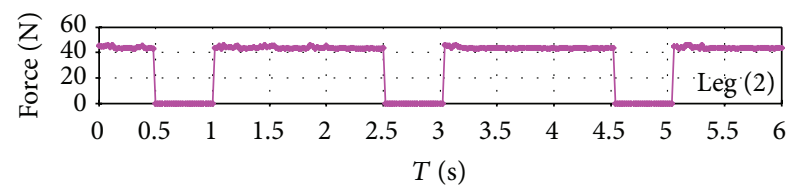

(b)

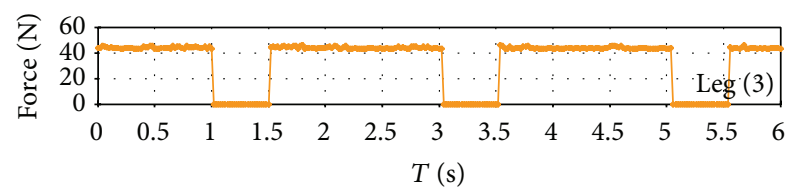

(c)

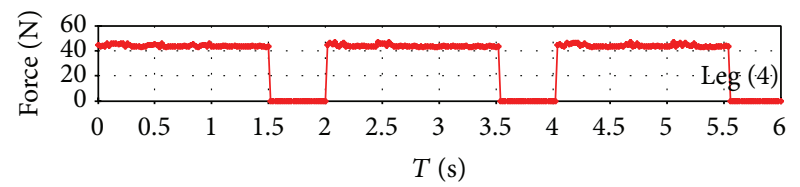

(d)

FIGURE 18: Foot supporting forces when duty cycle is 0.75 and the speed is 2 steps per second.

one-third of the system weight in the supporting phase. The time sequence of each leg force indicates that the walking paces are in a well-coordinated manner. This is ensured by the solid mechanical linkages between each leg and therefore 
it is a goal much easier to achieve than using individual servomotors on each leg joint.

A limitation of the system is that the duty cycle is fixed at 0.75 ; therefore, it is not very suitable for fast running, in which case the hanging phase might involve two legs lifted about the ground at the same time. However, this design does demonstrate a simple and reliable solution to perform fourleg walking with steady paces on flat terrain using much lower costs, and it may be applicable to some applications such as household robots or educational devices.

\section{Conclusion}

This study is focused on the walking mechanism design of a quadruped robot and it covers the studies in the following areas:

(i) introducing the basic concept of quadruped robot walking and analyzing typical quadruped walking characteristics, the kinematic bone structures, and step sequences;

(ii) investigating the mechanical theories of the quadruped robot walking mechanism and selecting the required control parameters;

(iii) investigating the kinematic principles of the quadruped robot walking mechanism and using hip joint cam, knee joint cam, and main cam to control the leg governing parameters;

(iv) building the three-dimensional model of the quadruped robot walking mechanism, developing a prototype machine, initially testing walking performance, and improving the system design;

(v) performing walking tests using the prototype machine and analyzing the force characteristics.

The cam drive bionic quadruped robot study is a long term research project, needing some theoretical breakthroughs and practical issues to be better handled in reality. In the later studies, more attention should be given to the optimal layout design of the mechanical components and to better stabilize the gravity center of the system for faster walking, as well as dynamics simulation of the system using virtual system simulation software MSC.ADAMS.

\section{Conflict of Interests}

The authors declare that there is no conflict of interests regarding the publication of this paper.

\section{Acknowledgments}

This work was supported by the Natural Science Foundation of Shandong Province, China (Grants nos. ZR2011EL038 and ZR2012CQ026), and a Project of Shandong Province Higher Educational Science and Technology Program (Grants nos. J11LD16 and J12LB63).

\section{References}

[1] R. B. McGhee and A. A. Frank, "On the stability properties of quadruped creeping gaits," Mathematical Biosciences, vol. 3, no. 1-2, pp. 331-351, 1968.

[2] V.S. Gurfinkel, E. V. Gurfinkel, A. Y. Shneider, E. A. Devjanin, A. V. Lensky, and L. G. Shtilman, "Walking robot with supervisory control," Mechanism and Machine Theory, vol. 16, no. 1, pp. 3136, 1981.

[3] S. Hirose, T. Masui, and H. Kikuchi, "TITANIII: a quadruped walking vehicle-its structure and basic characteristics," International Journal of Robotics Research, pp. 325-331, 1985.

[4] S. Hirose, K. Yoneda, K. Arai, and T. Ibe, "Design of prismatic quadruped walking vehicle TITAN VI," in Proceedings of the 5th International Conference on Advanced Robotics, pp. 723-728, Pisa, Italy, June 1991.

[5] S. Hirose, K. Yoneda, and H. Tsukagoshi, "TITAN VII: quadruped walking and manipulating robot on a steep slope," in Proceedings of the IEEE International Conference on Robotics and Automation, vol. 1, pp. 494-500, Albuquerque, NM, USA, April 1997.

[6] K. Kato and S. Hirose, "Development of the quadruped walking robot, TITAN-IX -mechanical design concept and application for the humanitarian de-mining robot," Advanced Robotics, vol. 15, no. 2, pp. 191-204, 2001.

[7] Y. Fukuoka, H. Kimura, Y. Hada, and K. Takase, "Adaptive dynamic walking of a quadruped robot "Tekken" on irregular terrain using a neural system model," in Proceedings of the IEEE International Conference on Robotics and Automation, pp. 20372042, Taipei, Taiwan, September 2003.

[8] M. Raibert, K. Blankespoor, G. Nelson et al., "The rough terrain quadruped robot," in Proceedings of the 17th International Federation of Automation Control, p. 10822, Seoul, Republic of Korea, 2008.

[9] L. Fei, Research about gait planning and balance control of quadruped robot [Ph.D. thesis], University of Science and Technology of China, Heifei, China, 2010.

[10] Y. Fukuoka, H. Kimura, and A. H. Cohen, "Adaptive dynamic walking of a quadruped robot on irregular terrain based on biological concepts," The International Journal of Robotics Research, vol. 22, no. 3-4, pp. 187-202, 2003.

[11] Z. G. Zhang, H. Kimura, and K. Takase, "Adaptive running of a quadruped robot using forced vibration and synchronization," Journal of Vibration and Control, vol. 12, no. 12, pp. 1361-1383, 2006.

[12] I. Poulakakis, E. Papadopoulos, and M. Buehler, "On the stability of the passive dynamics of quadrupedal running with a bounding gait," The International Journal of Robotics Research, vol. 25, no. 7, pp. 669-687, 2006.

[13] L. Ding, R. Wang, H. Feng, and J. Li, "Brief analysis of a BigDog quadruped robot," China Mechanical Engineering, vol. 23, no. 5, pp. 505-514, 2012.

[14] S. Qun, S. Chunlei, and L. Baolong, "Virtual design of the mechanical system of the quadruped robot and theory analysis of the wheel mechanism," Mechanical Design and Manufacture, vol. 46, no. 8, pp. 183-185, 2009.

[15] S. Chunlei, S. Qun, and L. Baolong, "Design and theory analysis for the stepped mechanism of the quadruped robot based on 
cam control drive," Mechanical Design and Manufacture, vol. 47, no. 3, pp. 168-170, 2010.

[16] S. Qun, S. Chunlei, and J. Zhijun, "Control system design of a flexible quadruped robot based on cam drive," Mechanical Design and Manufacture, vol. 44, no. 10, pp. 154-156, 2010. 

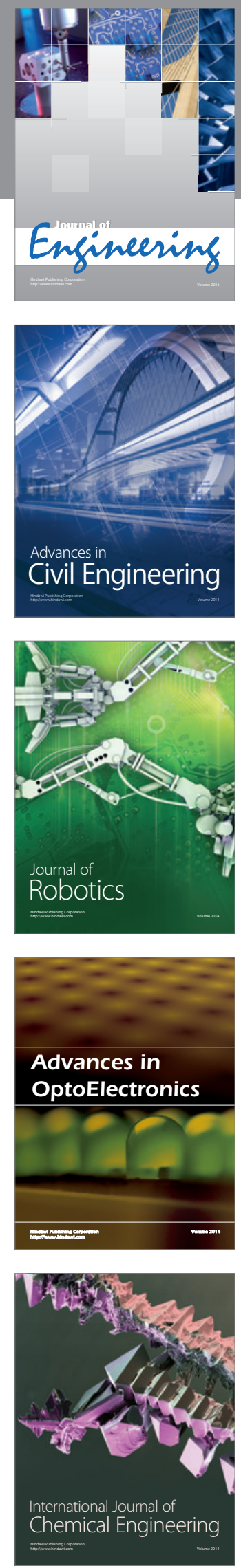

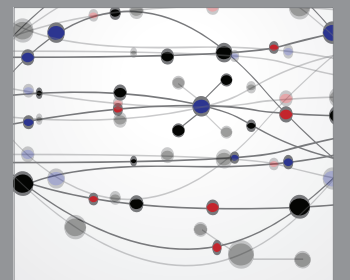

The Scientific World Journal
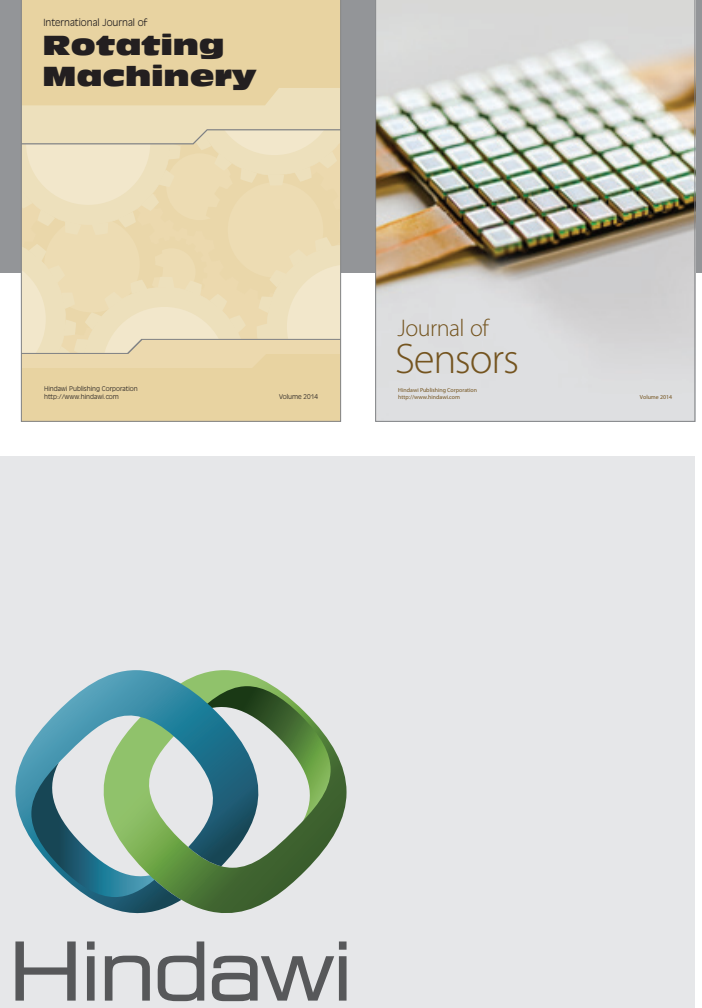

Submit your manuscripts at http://www.hindawi.com
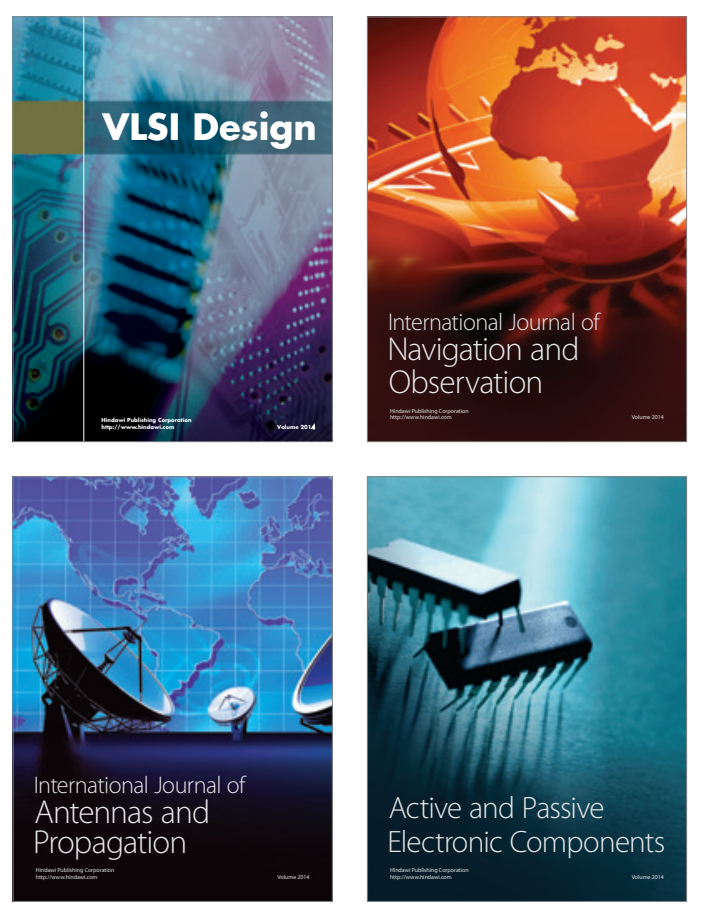
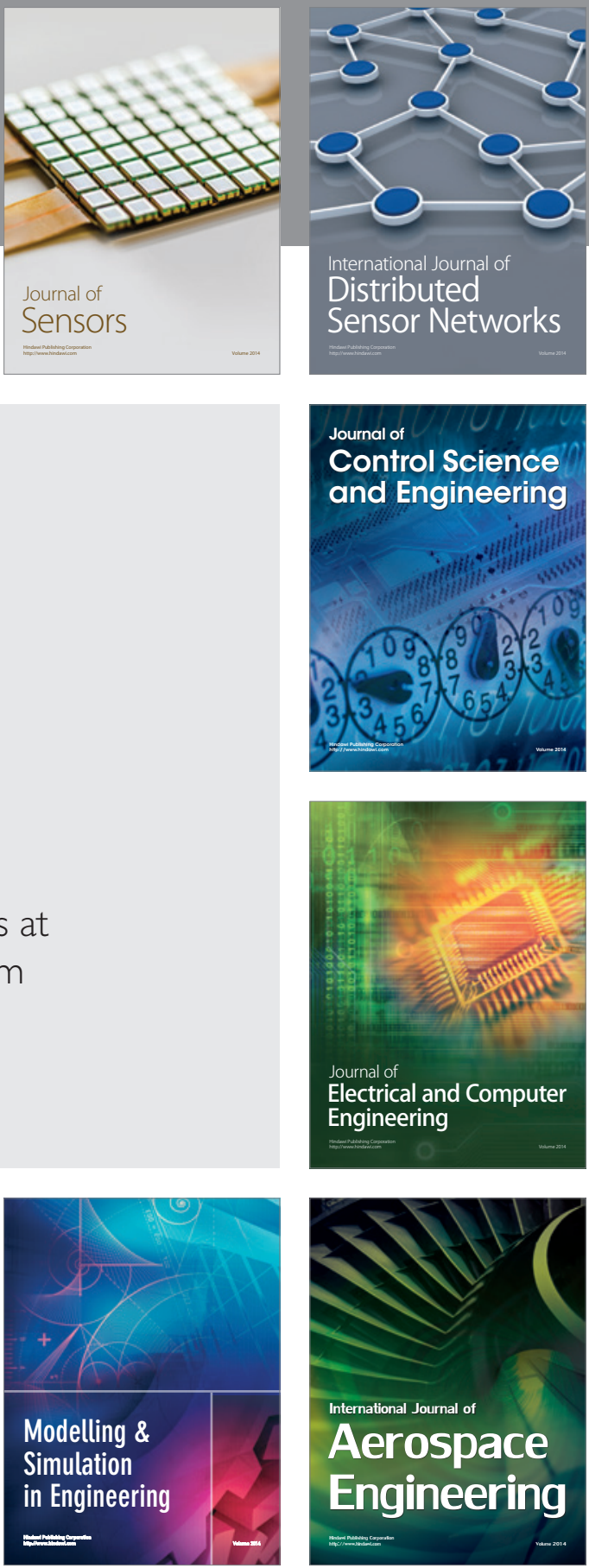

Journal of

Control Science

and Engineering
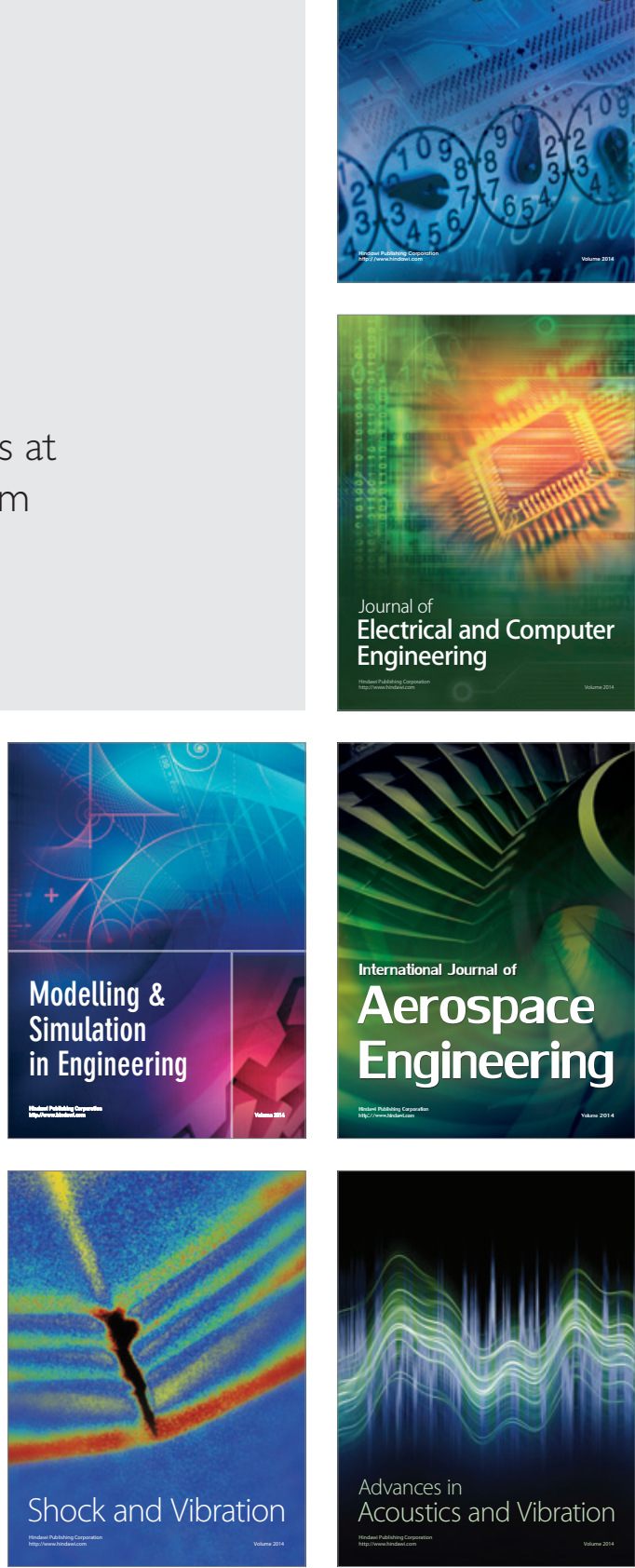